\title{
Magnetic response and electronic states of well defined Graphene/Fe/Ir(111) heterostructure
}

\author{
Claudia Cardoso $\odot,{ }^{1}$ Giulia Avvisati, ${ }^{2}$ Pierluigi Gargiani, ${ }^{3}$ Marco Sbroscia, ${ }^{2}$ Madan S. Jagadeesh, ${ }^{2}$ Carlo Mariani $\odot,{ }^{2}$ \\ Dario A. Leon ${ }^{\circ},{ }^{4,1}$ Daniele Varsano ${ }^{\circ},{ }^{1}$ Andrea Ferretti $\odot,{ }^{1}$ and Maria Grazia Betti ${ }^{2}{ }^{2}$ \\ ${ }^{1}$ Centro S3, CNR-Istituto Nanoscienze, I-41125 Modena, Italy \\ ${ }^{2}$ Dipartimento di Fisica, Università di Roma “La Sapienza”, I-00185 Roma, Italy \\ ${ }^{3}$ ALBA Synchrotron Light Source, Carrer de la Llum, 2-26 08290 Barcelona, Spain \\ ${ }^{4}$ Università di Modena e Reggio Emilia, I-41125 Modena, Italy
}

(Received 27 October 2020; accepted 22 December 2020; published 12 January 2021)

\begin{abstract}
We investigate a well defined heterostructure constituted by magnetic Fe layers sandwiched between graphene (Gr) and $\operatorname{Ir}(111)$. The challenging task to avoid Fe-C solubility and Fe-Ir intermixing has been achieved with atomic controlled Fe intercalation at moderate temperature below $500 \mathrm{~K}$. Upon intercalation of a single ordered Fe layer in registry with the Ir substrate, an intermixing of the Gr bands and Fe $d$ states breaks the symmetry of the Dirac cone, with a downshift in energy of the apex by about $3 \mathrm{eV}$, and well-localized $\mathrm{Fe}$ intermixed states induced in the energy region just below the Fermi level. First principles electronic structure calculations show a large spin splitting of the Fe states, resulting in a majority spin channel almost fully occupied and strongly hybridized with $\mathrm{Gr} \pi$ states. X-ray magnetic circular dichroism on the $\mathrm{Gr} / \mathrm{Fe} / \mathrm{Ir}$ heterostructure reveals an ordered spin configuration with a ferromagnetic response of Fe layer(s), with enhanced spin and orbital configurations with respect to the bcc-Fe bulk values. The magnetization switches from a perpendicular easy magnetization axis when the Fe single layer is lattice matched with the $\operatorname{Ir}(111)$ surface to a parallel one when the Fe thin film is almost commensurate with graphene.
\end{abstract}

DOI: 10.1103/PhysRevMaterials.5.014405

\section{INTRODUCTION}

Graphene can be a very promising spin channel material owing to its long spin-diffusion lengths of several micrometers [1], gate-tunable carrier concentration, and high electronic mobility [2]. Graphene coupled with ferromagnetic systems can open new perspectives when efficient injection of spin-polarized electrons can be achieved, as observed for a graphene membrane on $\mathrm{Co}(0001)$ [3]. It is well known that nearly flat epitaxial graphene of high structural quality can be formed on several magnetic $3 d$ metal substrates, like the lattice-matched $\mathrm{Ni}(111)$ [4-7] and $\mathrm{Co}(0001)$ [3,8-10] surfaces. It has been shown that a tiny magnetic moment arises on the carbon atoms, induced by the strong hybridization of graphene $\pi$ orbitals with $\mathrm{Ni}$ or Co $3 d$ states $[3,8,11,12]$. Furthermore, graphene grown on metals protects highly reactive magnetic surfaces and stabilizes them against oxidation [13-15]. Whereas recently a large research effort has been dedicated to investigate $\mathrm{Gr}-\mathrm{Ni}$ and $\mathrm{Gr}-\mathrm{Co}$ heterostructures, only a few experimental results for graphene grown on Fe surfaces are available [16-18], though iron is the most widespread transition metal, and the technology of passivated Fe films with a graphene membrane can be appealing for several reasons. Among them, the considerable price reduction in comparison with other transition metal substrates and, most importantly, its strong magnetic response.

The main hurdle for the formation of graphene on top of Fe surfaces is related to the rich $\mathrm{Fe}-\mathrm{C}$ phase diagram [19]. In fact, the high carbon diffusion coefficient and solubility in iron are detrimental for chemical vapor deposition processes, where a high annealing temperature is necessary to ensure high quality graphene on top of the metal surfaces. Thus, the epitaxial growth of a graphene membrane on a $\mathrm{Fe}$ film/single crystal is made difficult because of the formation of iron carbide, which is thermodynamically favored [19]. The epitaxial growth of graphene on $\mathrm{Fe}$ is also prevented because none of the bcc-Fe facets is lattice matched with graphene, at variance with close-packed surfaces of other $3 d$ metals, like Co and Ni. Recently, a detailed structural study has demonstrated that the $\mathrm{Fe}(110)$ face, with its distorted hexagonal symmetry, can be a good candidate, because of a partial match with the graphene lattice [16,17]. In that study, the substrate temperature was kept below $675 \mathrm{~K}$ to guarantee the formation of a graphene membrane on iron reducing the presence of iron carbides or segregation of diluted carbon from iron.

A more challenging strategy has been proposed exploiting $\mathrm{Fe}$ intercalation beneath graphene [20] as a viable route to overcome the hurdles to realize a direct growth on Fe surfaces. Intercalation of metals below Gr has proven to efficiently lead to the formation of atomically smooth metallic layer(s) $[8-10,12,21]$, in a layer-by-layer growth mode [22], where the graphene cover acts as a protective membrane of the confined epitaxial metallic layer(s) $[13,14]$. Recently, a scanning tunneling microscopy (STM) experiment demonstrates that successful Fe intercalation under graphene grown on $\operatorname{Ir}(111)$ can be obtained with the substrate temperature in the range between $450 \mathrm{~K}$ and $550 \mathrm{~K}$ [23], giving rise to a smooth $\mathrm{Fe}$ 
layer pseudomorphic with $\operatorname{Ir}(111)$ and a highly corrugated graphene membrane on top.

In this work, following this successful strategy for $\mathrm{Fe}$ growth, we have realized well-defined smooth Fe layers intercalated between $\mathrm{Gr}$ and $\operatorname{Ir}(111)$, preventing any alloying and $\mathrm{Fe}-\mathrm{C}$ intermixing. By means of a combined experimental and theoretical approach, we gain a detailed insight into the physical properties of the iron-intercalated $\mathrm{Gr} / \operatorname{Ir}(111)$ heterostructure. The sandwiched Fe layers present a redistribution of the minority and majority electronic density of states triggered by the spatial confinement and by the peculiar strained structural configurations, as predicted by first principles spin resolved electronic structure calculations and experimentally confirmed by angular resolved photoemission and $\mathrm{x}$-ray magnetic dichroism.

\section{METHODS}

Angular resolved photoelectron spectroscopy (ARPES) and low-energy electron-diffraction (LEED) experiments were carried out at the Nanostructure at Surfaces laboratory, Department of Physics, Sapienza University [24]. The ARPES apparatus is equipped with a Scienta SE200 multi-channel-plate electron analyzer and a monochromatic Gammadata VUV 5000 microwave He source, with main lines at $20.218 \mathrm{eV}\left(\mathrm{He} \mathrm{I}_{\alpha}\right)$ and $40.814 \mathrm{eV}\left(\mathrm{He} \mathrm{II}_{\alpha}\right)$ photon energies. The ARPES apparatus was set for an experimental energy and angular resolution of $16 \mathrm{meV}$ and $0.18^{\circ}$, respectively.

The $\mathrm{x}$-ray magnetic circular dichroism (XMCD) measurements were performed at the BOREAS beamline of the ALBA synchrotron radiation facility [25]. Data were taken in the total electron yield (TEY) mode, by measuring the drain current with respect to a clean gold grid, used for photon flux normalization. We used two different geometries, grazing incidence (GI) with $70^{\circ}$ incidence angle and normal incidence (NI), so to probe the magnetic signal along the easy and hard magnetic axes; further details are available in Ref. [26].

In all laboratories, the $\operatorname{Ir}(111)$ surface was cleaned by cycles of ion sputtering $\left(\mathrm{Ar}^{+}, 1.5-2.0 \mathrm{keV}\right)$ and annealing (1 minute at temperatures higher than $1300 \mathrm{~K}$ ). The quality of the obtained surface is confirmed by the sharp LEED pattern. The Gr layer was prepared by thermal decomposition of ethylene, by exposing the clean Ir surface to $5 \times 10^{-8}-2 \times 10^{-7} \mathrm{mbar}$ of $\mathrm{C}_{2} \mathrm{H}_{4}$ and annealing at around $1300-1320 \mathrm{~K}$. Metallic $\mathrm{Fe}$ was deposited, at $0.3 \AA / \mathrm{min}$ on the $\mathrm{Gr} / \operatorname{Ir}(111)$ surface kept at about $500 \mathrm{~K}$, in order to ensure a high quality of Fe layer(s) intercalated beneath Gr and to avoid any intermixing, that occurs at higher annealing temperature (600-800 K) [27]. The $\mathrm{C} 1 s$, Ir $4 f$, and $\mathrm{Fe} 2 p_{3 / 2}$ core levels as measured at the Superesca beamline of the Elettra synchrotron radiation facility, are presented in the Supplemental Material [28].

The completion of a single layer of $\mathrm{Fe}$ can be identified by following the evolution of the $\pi$ states of Gr in the ARPES valence band, as discussed in detail in the next sections. At the BOREAS beamline, the Fe density in the intercalated film was evaluated via its jump-edge ratio, previously calibrated with Auger electron spectroscopy, as reported in the Supporting Information of Ref. [22].

Density functional theory (DFT) simulations were carried out using the QUANTUM ESPRESSO package $[29,30]$ where wave functions are expanded in plane waves and pseudopotentials are used to account for the electron-ion interaction. We used the local density approximation (LDA) for the exchange-correlation potential, according to the PerdewZunger parametrization [31]. Similarly to our previous works [26,32], we simulated the Gr/1ML-Fe/Ir(111) interface considering the complete moiré induced periodicity by using a $9 \times 9$ supercell of $\operatorname{Ir}(111)$, corresponding to a $10 \times 10$ supercell of pristine Gr. The lattice parameters were obtained by relaxing Ir bulk at the LDA level using ultrasoft pseudopotentials (USPP), resulting in a Ir-Ir bond distance of $2.7048 \AA$ (corresponding to a hexagonal cell of $46.001 \mathrm{Bohr}$ radius for the moiré structure). In all the calculation we included four metallic layers ( 3 Ir plus one Fe layer or 4 Ir layers). In order to make the two sides of the slab inequivalent we added a layer of $\mathrm{H}$ atoms in one of the two sides. Atomic positions were then fully relaxed (except for the two bottom Ir layers and the $\mathrm{H}$ saturation layer) until ionic forces were smaller than 0.001 Ry/Bohr. For all the self-consistent calculations we used a $2 \times 2$ grid of $\mathbf{k}$ points, ultra soft pseudopotentials to model the electron-ion interaction and a kinetic energy cutoff of 30 and 300 Ry to represent Kohn-Sham wave functions and density, respectively.

Since LDA is known to underestimate the values of the orbital magnetic moments in transition metals [33], we have adopted a DFT $+U$ scheme [34], with a Hubbard $U$ parameter of $2 \mathrm{eV}$. In order to compare calculated band structures with ARPES data, we applied an unfolding procedure [35,36] to the computed bands of $\mathrm{Gr} / \mathrm{Fe} / \mathrm{Ir}(111)$ and $\mathrm{Gr} / \mathrm{Ir}(111)$. With this procedure, the band structure computed for the $10 \times 10$ supercell is mapped into the graphene $1 \times 1$ Brillouin zone by using the UNFOLD-X code [36]. In this way we obtain an effective band structure corresponding to the graphene unit cell. In this picture, the $\mathbf{k}$ dispersion is broadened by the break of the $1 \times 1$ translational symmetry induced by the $10 \times 10$ moiré pattern.

\section{RESULTS AND DISCUSSION}

\section{A. Intercalation and structural properties}

A single layer of iron, intercalated under Gr grown on $\operatorname{Ir}(111)$, induces a corrugation of the Gr membrane and preserves the periodicity of the moiré superstructure, superimposed to the hexagonal Gr lattice, as unraveled by the LEED patterns reported in Fig. 1(a)-1(b). The diffraction pattern of $\mathrm{Gr} / \operatorname{Ir}(111)$ [Fig. 1(a)] shows bright spots in a hexagonal pattern surrounded by satellites, consistent with the moiré superstructure caused by the lattice mismatch between $\mathrm{Gr}$ and Ir [37,38]. After Fe intercalation, the pattern is only slightly attenuated [Fig. 1(b)], thus the first Fe layer appears commensurate to the $\operatorname{Ir}(111)$ surface lattice. This evidence is corroborated by a recent work [12] where STM measurements show that a few monolayers (ML) of Fe intercalated under graphene arrange in registry with the hexagonal $\operatorname{Ir}(111)$ surface, with a corrugation of $1.3 \AA$, larger than the one measured for $\mathrm{Gr} / \operatorname{Ir}(111)$ [39].

We have further investigated the structural properties of this system by means of DFT at the LDA $+U$ level. The $\mathrm{Gr} / \mathrm{Fe} / \mathrm{Ir}(111)$ heterostructure was modeled as described in 
(a)

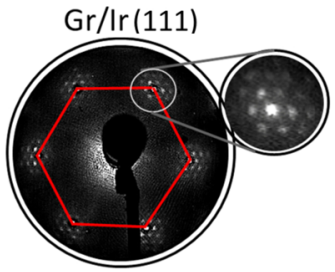

(b)

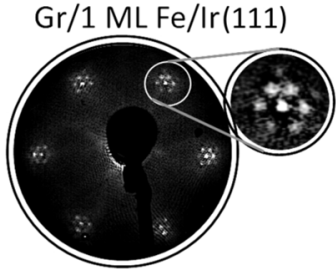

(c)

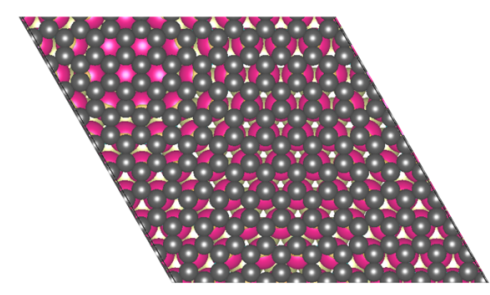

(d)

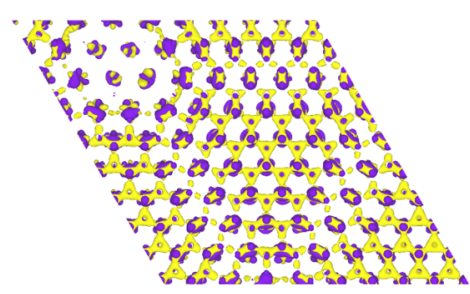

(e)

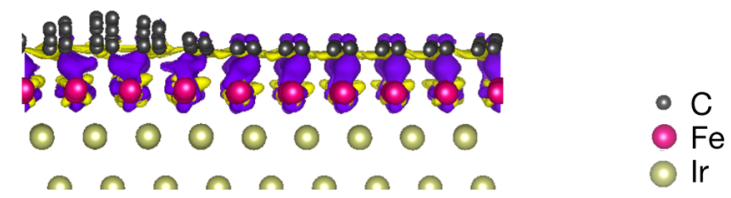

FIG. 1. Low energy electron diffraction (LEED) patterns (electron beam energy $140 \mathrm{eV}$ ) for (a) $\mathrm{Gr} / \mathrm{Ir}(111)$ and (b) Gr/1 ML Fe/Ir(111); (c) atomic structure as deduced by DFT, top view of the moiré pattern of $\mathrm{Gr} / \mathrm{Fe} / \mathrm{Ir}(111)$ with $\mathrm{C}$ atoms represented in gray, Fe in red, and $\mathrm{Ir}$ in cream; (d),(e) charge difference with respect to the free standing Gr and the pristine Fe/Ir(111) slab, computed with DFT: (d) top view of the charge difference isosurfaces (positive shown in purple, negative in yellow) and (e) side view of the $\mathrm{Gr} / \mathrm{Fe} / \mathrm{Ir}(111) \mathrm{structure}$ showing the graphene corrugation and charge difference isosurfaces, using the same colors described for the previous panels.

Sec. II. The calculations confirm the large corrugation of the graphene layer (1.44 $\AA$ ), with a minimum graphene-Fe interplanar distance of $1.85 \AA$ and a maximum of $3.29 \AA$ on the crests of the hills, as shown in Fig. 1(c). Previous DFT calculations [12] done at the PBE level including van der Waals interactions by using the DFT-D2 method [40,41] found a similar scenario, with slightly larger $\mathrm{Gr}-\mathrm{Fe}$ distances $(2.05$ and $3.33 \AA$ for valleys and hills, respectively).

The topography of the $\mathrm{Gr} / \mathrm{Fe} / \operatorname{Ir}(111)$ heterostructure is similar to the corrugated moiré superstructure observed for the $\mathrm{Gr} / \mathrm{Co} / \mathrm{Ir}(111)$ system [26], but very different from the structure reported for $\mathrm{Gr}$ directly grown on bcc-Fe(110) [16]. Previous STM measurements and DFT calculations performed at the LDA level, for $\mathrm{Gr} / \mathrm{bcc}-\mathrm{Fe}(110)$ point out the formation of a periodic corrugated pattern of the graphene layer parallel to the [001] direction of the substrate, consisting of a supercell of $7 \times 17$ graphene hexagons with a smaller corrugation of $0.6 / 0.9 \AA$ and only a small fraction of the $\mathrm{C}$ atoms considerably elevated over the Fe surface, thus making the entire graphene membrane strongly interacting with the metal substrate.

In the present case, the larger graphene corrugation modulates the $\mathrm{Gr} / \mathrm{Fe}$ interaction. This is illustrated by the charge difference computed for $\mathrm{Gr} / \mathrm{Fe} / \mathrm{Ir}(111)$ shown in Figs. 1(d) and 1(e). The excess of negative charge (yellow isosurface) is accumulated in the graphene membrane, donated by the $\mathrm{Fe}$ intercalated layer. The redistribution of charge is more pronounced in the valley regions and milder in the areas corresponding to the crests of the hills, corroborating a different strength of hybridization between graphene and Fe going from the valleys to the crests due to the increasing graphene-Fe distance. The periodicity of strongly and weakly bound regions in which covalent and van der Waals bonding dominate, respectively, induces a different balance in the charge transfer determined also by the registry with respect to the underlying $\mathrm{Fe}$ atoms.

Another signature of the similarity of the registry and graphene corrugation resulting from $\mathrm{Co}$ and $\mathrm{Fe}$ intercalated layers can be deduced by comparing the $\mathrm{C} 1 s$ core level photoemission data for $\mathrm{Gr} / \mathrm{Fe} / \mathrm{Ir}(111)$ (as reported in Supplemental Material [28]) and $\mathrm{Gr} / \mathrm{Co} / \operatorname{Ir}(111)$. In fact, there is a multicomponent $\mathrm{C} 1 s$ line shape for both systems, in which the two main features are assigned to $C$ atoms in the $\mathrm{Gr}$ layer weakly (on the crests) and strongly (in the valleys) bound to the $\mathrm{Co}$ and $\mathrm{Fe}$ layers [9,21,42]. In contrast, when graphene is grown directly on the bcc-Fe(110) surface, a single C $1 s$ core level component is present, explained by the presence of extended regions of graphene coupled to the $\mathrm{Fe}(110)$ substrate, even at the crests of the moiré superstructure $[16,17]$. For the graphene membrane directly grown on $\mathrm{Fe}(110)$, a small feature in the $\mathrm{C} 1 s$ core level has been detected at lower binding energy $(\mathrm{BE})$, linked to the presence of $\mathrm{Fe}$ carbide resulting from the $\mathrm{Fe}-\mathrm{C}$ solubility due to the high temperature reached during the chemical vapor deposition growth procedure $[16,17]$. The similarity of the $\mathrm{Fe}$ intercalation process under $\mathrm{Gr} / \mathrm{Ir}(111)$ with the $\mathrm{Gr} / \mathrm{Co} / \operatorname{Ir}(111)$ heterostructure and the absence of a C $1 s$ component due to the formation of iron carbide (see details in Supplemental Material [28]) demonstrate the successful strategy to intercalate Fe under graphene at low temperature, preventing any solubility into $\mathrm{C}$.

The corrugation of the Gr membrane discussed so far is attenuated when the number of Fe intercalated layers increases above a few ML, with a reduction of the brightness of the superstructure LEED spots, as reported in Figs. 2(a)-2(c). At $7 \mathrm{ML}$, we observe the formation of a $\mathrm{Fe}$ film with a lattice constant smaller than $\operatorname{Ir}(111)$ and therefore closer to the graphene structural parameters. However, even when the strain induced by the $\operatorname{Ir}(111)$ surface is released, the Fe film remains slightly incommensurate with graphene, as shown in Fig. 2(a). This is in contrast with the case of Co intercalation beneath graphene on $\operatorname{Ir}(111)$ : when the Co film is formed, it recovers its hpc lattice constant and becomes commensurate with the graphene layer [9,42]. Indeed, while the hcp $\mathrm{Co}(0001)$ surface lattice parameter $(2.50 \AA)$ is comparable with the $2.46 \AA$ of $\mathrm{Gr}$, none of the bcc-Fe faces is lattice matched with graphene. In fact, graphene directly grown on 
(a)

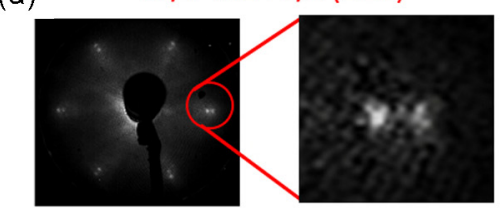

(b)

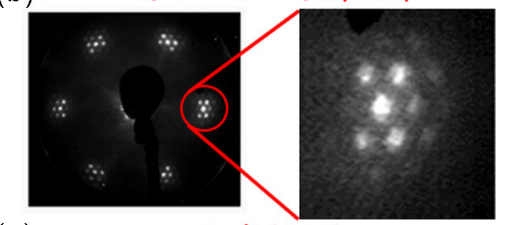

(c)
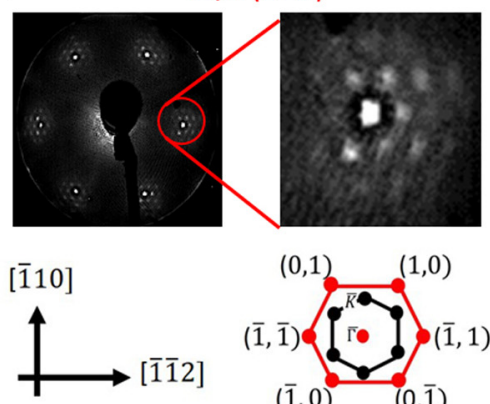

[1̄12]

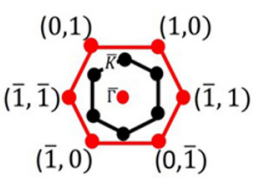

(d)

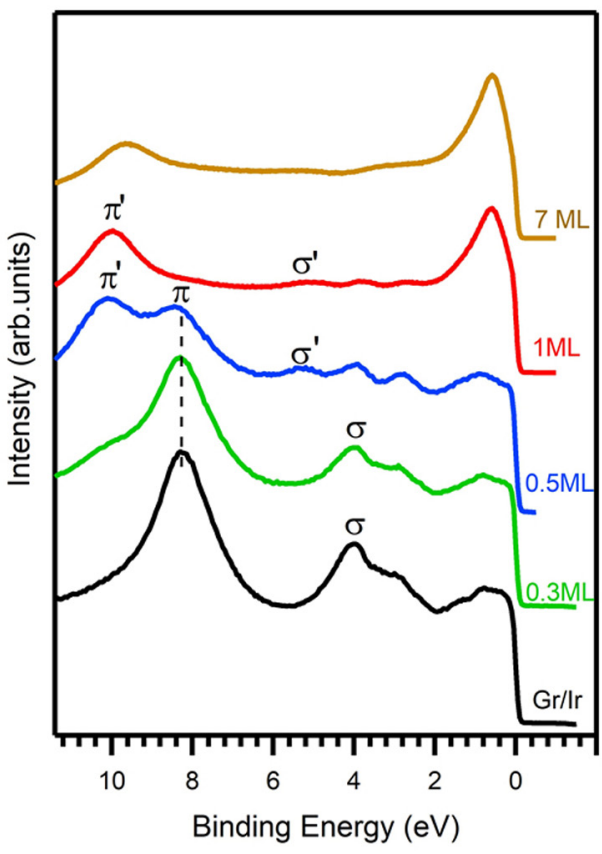

(e)

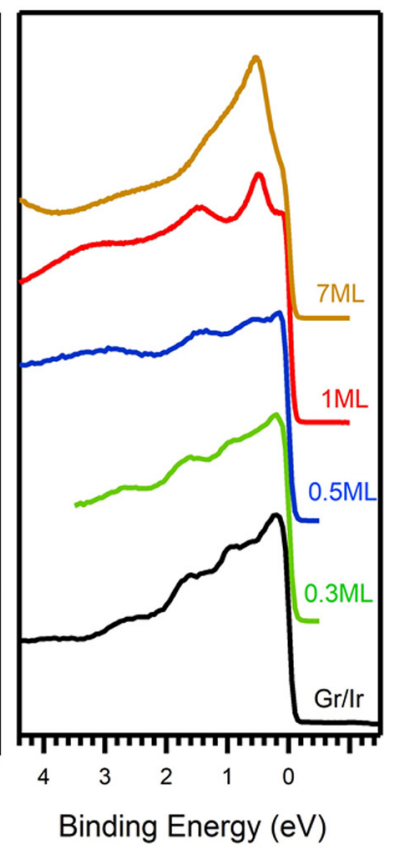

FIG. 2. LEED patterns (electron beam energy $140 \mathrm{eV}$ ) and zoomed pattern around the (10) diffraction spot for Gr/7ML Fe/Ir(111) (a), $\mathrm{Gr} / 0.5 \mathrm{ML} \mathrm{Fe} / \operatorname{Ir}(111)$ (b) and $\mathrm{Gr} / \operatorname{Ir}(111)$ (c); below: sketch of the main symmetry directions and of the diffraction pattern of the $\operatorname{Ir}(111)$ surface (red dots); the Surface Brillouin Zone (SBZ) is shown in black. Photoemission energy distribution curves (EDC) taken at 40.814 eV $\left(\mathrm{He} \mathrm{II}_{\alpha}\right)$; angular integrated spectra around $\Gamma(\mathrm{d})$ and $K(\mathrm{e})$ points of the SBZ for different thickness of the intercalated Fe layer between $\mathrm{Gr}$ and Ir.

bcc-Fe(110) presents a distorted hexagonal symmetry with only a partial match with the graphene lattice, as deduced by STM [16]. In our case, the Fe intercalated multilayer has a novel strained structural configuration with a $8 \%$ mismatch with respect to the graphene lattice constant (see details of the LEED spot intensity analysis in the Supplemental Material [28]).

Importantly, the magnetic response of Fe films is extremely sensitive to tiny variations of the structural configuration [43], and the strained lattice of the Fe film intercalated under graphene can give rise to an altered distribution of the electronic majority and minority states and to different spin and orbital configurations with respect to the bulk reference [44].

\section{B. Electronic structure}

A deeper insight into the interaction between $\mathrm{Gr}$ and the Fe intercalated layer(s) can be unveiled by the electronic spectral density deduced by angular resolved photoelectron spectroscopy of the $\mathrm{Gr} / \mathrm{Fe} / \operatorname{Ir}(111)$ heterostructure, compared with $a b$ initio theoretical predictions of the electronic density of states (DOS) and band structure calculated by DFT.

\section{Experimental data}

The photoemission spectral density as a function of Fe intercalation thickness is reported in Figs. 2(d) and 2(e), at the $\Gamma$ and $K$ points of the surface Brillouin zone (SBZ), respectively. The $\mathrm{Gr} / \operatorname{Ir}(111)$ valence band at the $\Gamma$ and $K$ points presents the expected electronic spectral density $[45,46]$ whereas the intercalated system shows three new features when compared with the bare $\mathrm{Gr} / \mathrm{Ir}(111)$ : (i) the appearance of a peak at $10 \mathrm{eV}$ and the disappearance of the one related to the $\pi$ bands of bare $\mathrm{Gr} / \mathrm{Ir}(111)$ at $8 \mathrm{eV}$ at the $\Gamma$ point; (ii) the quenching of the intensity from the Ir surface states close to the Fermi level; (iii) the emergence of extra spectral density in the low binding energy region.

Concerning (i), the intensity of the peak at $8 \mathrm{eV} \mathrm{BE}$ at $\Gamma$, corresponding to the bottom of the $\pi$ band for bare $\mathrm{Gr} / \mathrm{Ir}(111)$, decreases with increasing Fe thickness. The peak emerging at $10.0 \mathrm{eV} \mathrm{BE}$ can be associated to the shifted $\pi$ band, due to the interaction of $\mathrm{Gr}$ with the intercalated $\mathrm{Fe}$ layer. An interacting $\pi$ band has also been observed for Co and $\mathrm{Ni}$ layers sandwiched between $\mathrm{Gr}$ and $\operatorname{Ir}[5,7,9,10]$. The presence of two features associated with the $\pi$ bands, with opposite intensity behavior before the completion of the first Fe ML, demonstrates the coexistence of bare Gr/Ir regions and regions of intercalated $\mathrm{Fe}$ atoms, up to the formation of a smooth Fe single layer, when the peak at $8 \mathrm{eV}$ (bare $\mathrm{Gr} / \mathrm{Ir}$ ) is eventually quenched.

Furthermore, (ii) the progressive intensity lowering of the $\operatorname{Ir}(111)$ surface states in the energy region $0-3 \mathrm{eV}$ BE, upon Fe intercalation, without any energy shift, suggests the absence of Fe-Ir intermixing observed at higher intercalation temperatures [23,27]. The emergence of a spectral density (iii) at low binding energy close to the Fermi level can be ascribed to electronic states mainly localized in the $\mathrm{Fe}$ layer, since their intensity definitely raises at increasing $\mathrm{Fe}$ thickness, as can be clearly observed in the spectral density 

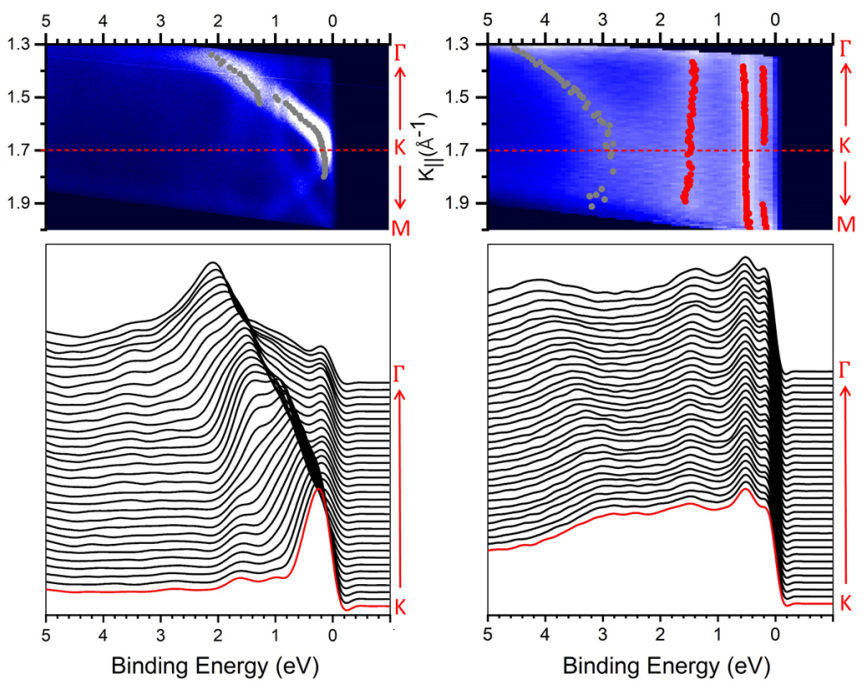

FIG. 3. Angular resolved photoelectron spectroscopy $\mathrm{h} v=$ $40.814 \mathrm{eV}$ spectra of $\mathrm{Gr} / \mathrm{Ir}(111)$ (left panels) and $\mathrm{Gr} / 1 \mathrm{ML}$ $\mathrm{Fe} / \mathrm{Ir}(111)$ (right panels), around the $K$ point of the SBZ. Electronic band structure $\mathrm{vs}_{\text {// }}$ as intensity plot (upper panels); spectral density plotted as sequential EDC curves from the $K$ point towards $\Gamma$ (bottom panels).

of $7 \mathrm{ML}$ Fe intercalated under graphene [orange curve in Fig. 2(e)].

A clearer assignment of the electronic states due to the FeGr interaction process can be derived from the electronic state dispersion. The ARPES data around the $K$ point of the SBZ for $\mathrm{Gr} / \mathrm{Ir}(111)$ and $\mathrm{Gr} / 1 \mathrm{ML} \mathrm{Fe} / \mathrm{Ir}(111)$ are shown in Fig. 3 (left and right panels, respectively). Considering the energy region closer to the Fermi level, three localized Fe states can be identified at about $0.2 \mathrm{eV}, 0.5 \mathrm{eV}$, and $1.5 \mathrm{eV} \mathrm{BE}$, and found to be only slightly dispersing over the whole SBZ (Fig. 3 right panel), as expected for a confined $\mathrm{Fe}$ film. Inspecting the spectra at higher binding energies, other Fe-related states are observed in the energy region of 3-4 eV BE, resonant with the graphene $\pi$-band states. Besides the presence of these localized Fe states close to the Fermi level, the most evident consequence of Fe intercalation under graphene is the downshift of the Dirac cone at the $K$ point of the SBZ, similarly to the case of $\mathrm{Gr} / \mathrm{Co} / \operatorname{Ir}(111)$ [9]. In fact, the $\pi$ band, as deduced by the EDCs (Fig. 3), is broadened and less defined after intercalation and there is a coexistence of other Fe related states in this energy region (3-4 eV). The position of the Dirac cone vertex (at $\mathrm{K}$ ) is downshifted by $3 \mathrm{eV}$, while the bottom of the $\pi$ band is less shifted at $\Gamma$. It is worth noting that the Dirac cone is shifted by $2 \mathrm{eV}$ towards higher binding energy also for graphene directly grown on $\mathrm{Fe}(110)$ [18], suggesting a weaker interaction in that case.

\section{Theory}

The measured ARPES data are complemented by DFT calculations, as shown in Fig. 4, where the electronic structures computed for $\mathrm{Gr} / \mathrm{Ir}(111)$ and $\mathrm{Gr} / \mathrm{Fe} / \mathrm{Ir}(111)$ are unfolded and mapped into the $1 \times 1$ graphene Brillouin zone along the $\Gamma-K$ direction, as described in Sec. II. Projected DOS (and projected bands in Supplemental Material [28]) are also provided to complement the band information. The bands obtained for $\mathrm{Gr} / \mathrm{Ir}(111)$ are in good agreement with existing literature, as from, e.g., Ref. [47]. Concerning the Fe intercalated system, as also observed in the experiments, the $\operatorname{Gr} \pi$ and $\sigma$ bands are shifted to higher binding energies by the effect of $\mathrm{Fe}$ intercalation (i). While for $\mathrm{Gr} / \mathrm{Ir}(111)$ the $\pi$ bands are clearly recognizable, in the case of $\mathrm{Gr} / \mathrm{Fe} / \mathrm{Ir}(111)$ they are strongly hybridized with $\mathrm{Fe}$ states above $-6 \mathrm{eV}$ and the upper part of the cone is identifiable only for the minority spin states. The bottom of the $\pi$ bands at $\Gamma$ moves from about $-7.5 \mathrm{eV}$ in $\mathrm{Gr} / \mathrm{Ir}$ to about $-10.0 \mathrm{eV}$ in $\mathrm{Gr} / \mathrm{Fe} / \mathrm{Ir}$ (see Fig. S4 in Supplemental Material [28]), with an overall downshift of $\sim 2.5 \mathrm{eV}$, in quite good agreement with the experimental findings. (a)

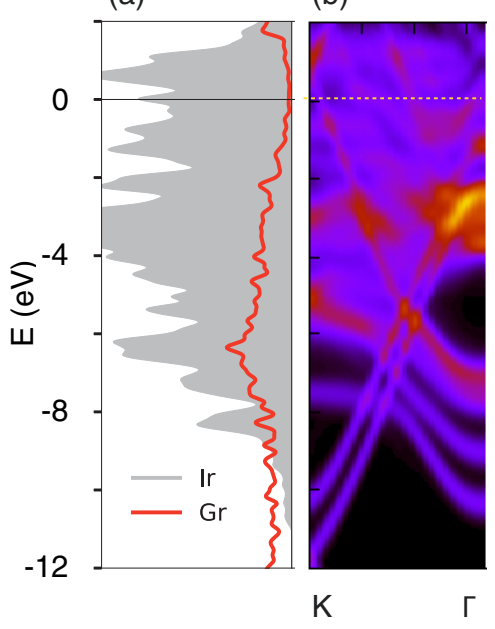

(c)

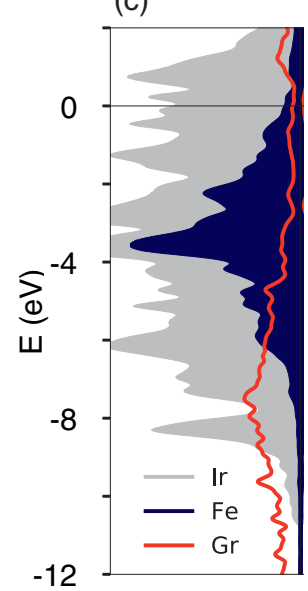

(d)

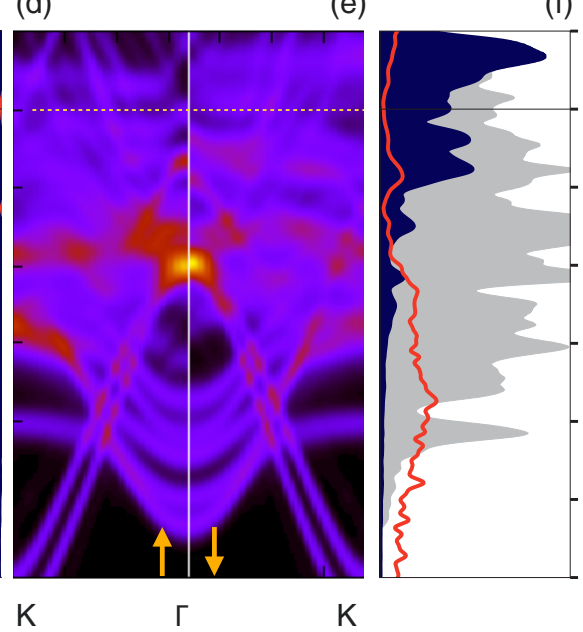

FIG. 4. Band structure and projected density of states (pDOS) computed within DFT for $\mathrm{Gr} / \mathrm{Ir}(111)$ and $\mathrm{Gr} / 1 \mathrm{ML} \mathrm{Fe} / \mathrm{Ir}(111)$ : (a) $\mathrm{Gr} / \operatorname{Ir}(111)$ DOS projected on $\mathrm{C}$ and Ir atomic orbitals and (b) $\mathrm{Gr} / \operatorname{Ir}(111)$ band structure, unfolded on the $1 \times 1$ graphene unit cell as described in the main text; (c) Gr/Fe/Ir(111) majority spin DOS projected on C, Fe, and Ir atomic orbitals; (d) majority and (e) minority spin band structure, unfolded on the $1 \times 1$ graphene unit cell; (f) $\mathrm{Gr} / \mathrm{Fe} / \mathrm{Ir}(111)$ minority spin pDOS. 
Similarly, the $\sigma$ bands of graphene undergo a downshift by about $-1.5 \mathrm{eV}$ at both $\Gamma$ (going from -2.5 to $-4 \mathrm{eV}$ ) and $K$ (from -10.5 to $-12 \mathrm{eV}$ ). While the experimental offset of these features is found at larger binding energies [namely $4.0 \mathrm{eV}$ and $5.5 \mathrm{eV}$ as marked by $\sigma$ and $\sigma^{\prime}$ in Fig. 2(d)], the downshift is quite consistent with the calculations [48].

In order to identify Fe-related states, we consider the projected DOS, shown in Figs. 4(c) and 4(f), where a strong spin splitting of the Fe components is observed (see discussion below). For instance, a large peak of the Fe pDOS is found in the energy region of 3-4 eV BE, in good agreement with the experimental data, and mainly located in the majority spin channel. Such a peak then overlaps with the $\pi$ bands of $\mathrm{Gr}$ disrupting the Dirac cone for majority states. In contrast, the Dirac cone is still faintly visible in the minority spin bands at about 2.5-3 eV. In both spin channels the pDOS reveals overlapping peaks due to Fe and graphene states, suggesting that the shadowing of the vertex of the Dirac cone observed in the experimental photoemission data is clearly induced by the hybridization of the graphene $\pi$ states with the Fe $d$ majority states. Furthermore, the spin resolved DOS in the energy region closer to the Fermi level shows that the main Fe-related peak of the minority spin states lies above the Fermi level, with smaller peaks between $-2 \mathrm{eV}$ and the Fermi energy, in good agreement with the experimental observation (iii). Overall, this picture is further confirmed by the projected unfolded bands provided in Fig. S4 of Supplemental Material [28].

\section{Magnetic properties and spin and orbital configuration of the Fe layer}

In contrast with Fe grown on $\operatorname{Ir}(111)$, for which the loss of inversion symmetry at the interface of the magnetic layer and substrate stabilizes different skyrmion lattices depending on the Fe/Ir stacking [49-53], when a Fe monolayer is intercalated between $\mathrm{Gr}$ and $\operatorname{Ir}(111)$, the heterostructure exhibits a ferromagnetic order with an out-of-plane easy magnetization axis [12]. For this reason, and based on the experimental evidence described below, in the present DFT calculations we have considered only collinear magnetic configurations, excluding in this way skyrmions or spin spiral textures.

The pDOS computed for the $\mathrm{Gr} / \mathrm{Fe} / \mathrm{Ir}(111)$ heterostructure shows a large spin split of the Fe states, with the maxima of the two spin pDOS almost $5 \mathrm{eV}$ apart. In particular, the majority spin states are almost fully occupied while the largest peak of the minority spin states is empty, which results in an average Fe-magnetic moment of $2.2 \mu_{B}$ /atom. The computed ground state magnetic configuration is ferromagnetic even if the Fe magnetic moment shows a modulation over different sites ranging from 2.0 to $2.7 \mu_{B}$ /atom, in good agreement with previous calculation [54]. The modulation is determined by the graphene layer, with the Fe atoms located below the graphene crests, i.e., with larger Fe-C distances, having the largest values. Concerning graphene, the $\mathrm{C}$ magnetic moments are nonzero but quite small (at the LDA $+U$ level ranging from -0.02 to $0.01 \mu_{B}$ /atom), similarly to what was reported for $\mathrm{Gr} / \mathrm{Co} / \operatorname{Ir}(111)[10,26]$, with a distance- and sublatticedependent modulation.
Overall, we find that the Fe monolayer displays a clear spin splitting, as an effect of the band narrowing and subsequent increased number of majority electrons. This is confirmed by the strong localization of the Fe-induced electronic states observed in the photoemission spectral density. It is interesting to point out that a different scenario is found for the $\mathrm{Gr} / \mathrm{Co} / \mathrm{Ir}(111)$ interface [10] (see Supplemental Material [28] for a detailed comparison with $\mathrm{Gr} / \mathrm{Co} / \mathrm{Ir}(111) \mathrm{pDOS}$ ) where Co spectral weight in the minority channel is shifted at lower energies leading to a spin split significantly smaller than in the present case.

The strong ferromagnetic behavior is confirmed by the $\mathrm{X}$-ray absorption spectra and the XMCD at the $\mathrm{L}_{2,3}$ edges for 1.4 ML and 7 ML of Fe sandwiched between $\mathrm{Gr}$ and $\operatorname{Ir}(111)$, shown in Fig. 5. XAS are acquired in remanence conditions with circularly polarized radiation and with photons impinging normal (left panel) and grazing (right panel) to the surface. The XMCD spectra are obtained as the difference between the absorption edges acquired with left- and rightcircularly polarized radiation. The higher dichroic response with photon impinging at normal incidence (left panel) reveals an out-of-plane magnetic anisotropy of the Fe layer when it is pseudomorphic to the $\operatorname{Ir}(111)$ surface, with a stretched Fe-Fe distance with respect the bulk bcc(110) or (111) surfaces. The higher dichroic response for $\mathrm{Fe}_{2,3} \mathrm{XMCD}$ at grazing incidence (right panel) for the thicker intercalated Fe film unveils a switch of the easy magnetization axis from perpendicular to the surface to in-plane. This is consistent with the magnetic response of heterostructures of single $\mathrm{Co}$ or $\mathrm{Fe}_{x} \mathrm{Co}_{1-x}$ layers on $\operatorname{Ir}(111)$ covered with a Gr membrane [21,26].

The spin and the orbital moments at the Fe site, as deduced via XMCD sum rules, show a doubled $L / S_{\text {eff }}$ ratio ( $S_{\text {eff }}=S+7 D$ and $D$ is the dipolar moment [55,56]), with respect to the bulk element $(0.11 \pm 0.01$ for a Fe single layer and $0.09 \pm 0.01$ for the $7 \mathrm{ML}$ ). These experimental results, related only to the intensity of the dichroic signal and independent on the number of $3 d$ holes, suggest an enhanced magnetic response due to the redistribution of the majority spin states in the conduction Fe bands. The evaluated average total moment is $2.1 \pm 0.2 \mu_{B}$ /atom for $7 \mathrm{ML}$ of Fe intercalated under $\mathrm{Gr}$, in fair agreement with $2.2 \mu_{B}$ /atom (spin moment), as deduced by the DFT predictions. The orbital and spin moment for a single layer of $\mathrm{Fe}$, where interface effects can play a role, shows a comparable magnetic response, as deduced by the similar dichroic signals reported in Fig. 5. As mentioned above, the pseudomorphic hexagonal Fe single layer grown directly on $\operatorname{Ir}(111)$ presents a complex magnetic structure with skyrmionic spin spiral textures stabilized by the $3 d-5 d$ hybridization between Fe and Ir. [50-53]. On the other hand, other magnetic materials like Europium have been successfully intercalated underneath graphene on the same Ir(111) surface [57] and, depending on its coverage, Eu displays either a paramagnetic or a ferromagnetic behavior [58]. The clear magnetic dichroism response of the single Fe layer intercalated under graphene suggests a different spin configuration. Furthermore, this confined single Fe intercalated layer, with stretched Fe-Fe distances, presents spin and orbital configurations similar to those of the thin Fe intercalated film, where the influence of the Fe-Ir interface is completely released. 
XMCD Fe $\mathrm{L}_{2,3}$ edge

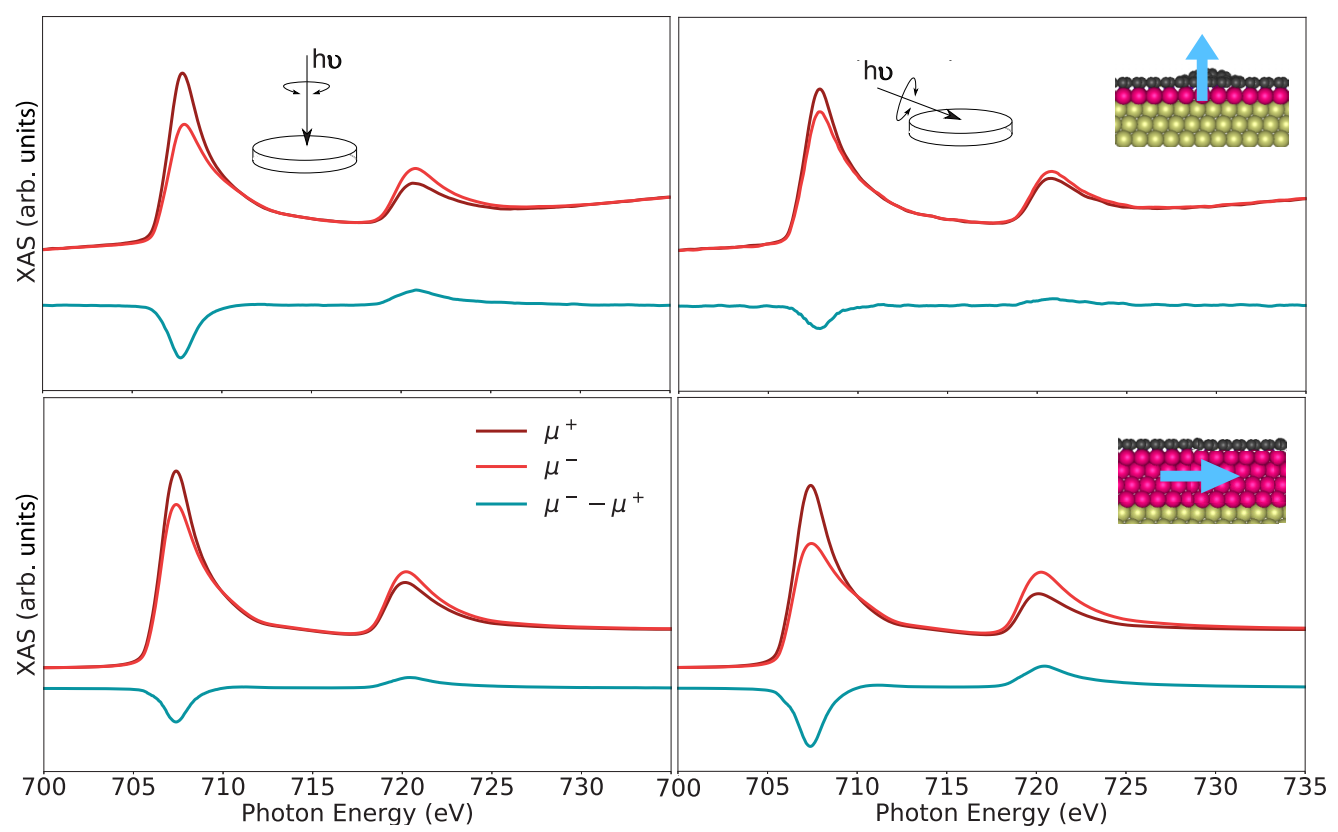

FIG. 5. XMCD spectra of $\mathrm{Fe} \mathrm{L}_{2,3}$ absorption edges for Gr/1.4 ML Fe/Ir(111) (top) and Gr/7ML Fe/Ir(111) (bottom) acquired in remanence at RT in normal (left) and grazing (right) incidence geometries (see sketches for the experimental geometry). The easy magnetization direction switching from perpendicular (top) to parallel (bottom) to the surface plane is indicated by the blue arrows.

\section{CONCLUSIONS}

The strategy to sandwich the Fe layer beneath the graphene membrane with an intercalation process at moderate temperature $(500 \mathrm{~K})$ prevents any alloying, and the absence of any hallmark of Fe-Ir and $\mathrm{Fe}-\mathrm{C}$ intermixing proves the formation of a well-defined homogeneous $\mathrm{Fe}$ layer in registry with the $\operatorname{Ir}(111)$ surface. Such a single layer of Fe, protected by the graphene membrane, induces a downshift in energy and a symmetry breaking of the Dirac cone due to the interaction between $\mathrm{Gr}$ and Fe majority states resonant in the energy region of the vertex of the cone. The redistribution of the spin resolved Fe pDOS with a narrowing of the $\mathrm{Fe}$ bands and a larger spin splitting between majority (fully occupied) and minority states (almost empty) of the Fe states associated with increased total Fe magnetic moment influences the magnetic response of the Fe intercalated layer(s).

In contrast to the case of direct growth of Fe on $\operatorname{Ir}(111)$, where, despite the large Fe magnetic moment, a noncollinear magnetic order has been observed [49-52], the Fe layer with graphene on top reveals a ferromagnetic long range order with spin and orbital moments that are higher than the ones found for the bulk phases. The graphene top layer acts not only as a protective membrane but also allows for a stable ferromagnetic configuration, counteracting the effect of Ir substrate. The concomitant dimensionality reduction with a narrowing of the $d$ bands and a reduced superimposition between the spin-split majority and minority Fe bands further contributes to the transition of a single Fe layer (or few layers of $\mathrm{Fe}$ ), from weak to strong ferromagnet, when intercalated beneath graphene. These $3 d$ confined layers protected by a graphene membrane with a novel structural configuration with respect to the bulk lattice arrangement offer a powerful playground to tune their electronic structure and magnetic state for magnetic/spintronic devices.

\section{ACKNOWLEDGMENTS}

The authors thank the experimental assistance of BOREAS beamline staff at ALBA, of SuperESCA beamline staff at Elettra, and of Alessio Vegliante in LOTUS laboratory in Rome. G.A. and M.G.B. acknowledge support from translational access CalipsoPlus funding. C.C., D.V., A.F., and D.A.L. acknowledge partially support from the MaX-MAterials design at the eXascale-Centre of Excellence, funded by the European Union program H2020-INFRAEDI-2018-1 (Grant No. 824143). They also thank SUPER (Supercomputing Unified Platform-Emilia-Romagna) from Emilia-Romagna POR-FESR 2014-2020 regional funds. The work was partially supported by PRIN FERMAT (2017KFY7XF) from Italian Ministry MIUR and by Sapienza Ateneo funds. Computational time on the Marconi100 machine at CINECA was provided by the Italian ISCRA program.
[1] N. Tombros, C. Jozsa, M. Popinciuc, H. T. Jonkman, and B. J. van Wees, Nature (London) 448, 571 (2007).
[2] W. Han, R. Kawakami, M. Gmitra, and J. Fabian, Nat. Nanotechnol. 9, 794 (2014). 
[3] D. Usachov, A. Fedorov, M. M. Otrokov, A. Chikina, O. Vilkov, A. Petukhov, A. G. Rybkin, Y. M. Koroteev, E. V. Chulkov, V. K. Adamchuk, A. Grüneis, C. Laubschat, and D. V. Vyalikh, Nano Lett. 15, 2396 (2015).

[4] L. V. Dzemiantsova, M. Karolak, F. Lofink, A. Kubetzka, B. Sachs, K. von Bergmann, S. Hankemeier, T. O. Wehling, R. Frömter, H. P. Oepen, A. I. Lichtenstein, and R. Wiesendanger, Phys. Rev. B 84, 205431 (2011).

[5] D. Pacilè, P. Leicht, M. Papagno, P. M. Sheverdyaeva, P. Moras, C. Carbone, K. Krausert, L. Zielke, M. Fonin, Y. S. Dedkov, F. Mittendorfer, J. Doppler, A. Garhofer, and J. Redinger, Phys. Rev. B 87, 035420 (2013).

[6] A. Varykhalov, D. Marchenko, J. Sánchez-Barriga, M. R. Scholz, B. Verberck, B. Trauzettel, T. O. Wehling, C. Carbone, and O. Rader, Phys. Rev. X 2, 041017 (2012).

[7] L. Massimi, S. Lisi, D. Pacilè, C. Mariani, and M. G. Betti, Beilstein J. Nanotech. 5, 308 (2014).

[8] R. Decker, J. Brede, N. Atodiresei, V. Caciuc, S. Blügel, and R. Wiesendanger, Phys. Rev. B 87, 041403(R) (2013).

[9] D. Pacilè, S. Lisi, I. Di Bernardo, M. Papagno, L. Ferrari, M. Pisarra, M. Caputo, S. K. Mahatha, P. M. Sheverdyaeva, P. Moras, P. Lacovig, S. Lizzit, A. Baraldi, M. G. Betti, and C. Carbone, Phys. Rev. B 90, 195446 (2014).

[10] H. Vita, S. Böttcher, P. Leicht, K. Horn, A. B. Shick, and F. Máca, Phys. Rev. B 90, 165432 (2014).

[11] M. Weser, Y. Rehder, K. Horn, M. Sicot, M. Fonin, A. B. Preobrajenski, E. N. Voloshina, E. Goering, and Y. S. Dedkov, Appl. Phys. Lett. 96, 012504 (2010).

[12] R. Decker, M. Bazarnik, N. Atodiresei, V. Caciuc, S. Blügel, and R. Wiesendanger, J. Phys.: Condens. Matter 26, 394004 (2014).

[13] J. Coraux, A. T. N'Diaye, N. Rougemaille, C. Vo-Van, A. Kimouche, H.-X. Yang, M. Chshiev, N. Bendiab, O. Fruchart, and A. K. Schmid, J. Phys. Chem. Lett. 3, 2059 (2012).

[14] M. Cattelan, G. W. Peng, E. Cavaliere, L. Artiglia, A. Barinov, L. T. Roling, M. Favaro, I. Píš, S. Nappini, E. Magnano, F. Bondino, L. Gavioli, S. Agnoli, M. Mavrikakis, and G. Granozzi, Nanoscale 7, 2450 (2015).

[15] A. Lodesani, A. Picone, A. Brambilla, D. Giannotti, M. S. Jagadeesh, A. Calloni, G. Bussetti, G. Berti, M. Zani, M. Finazzi et al., ACS nano 13, 4361 (2019).

[16] N. A. Vinogradov, A. A. Zakharov, V. Kocevski, J. Rusz, K. A. Simonov, O. Eriksson, A. Mikkelsen, E. Lundgren, A. S. Vinogradov, N. Mårtensson, and A. B. Preobrajenski, Phys. Rev. Lett. 109, 026101 (2012).

[17] N. Vinogradov, K. Simonov, A. Generalov, J. Drnec, F. Carlá, A. Vinogradov, A. Preobrajenski, N. Mårtensson, and R. Felici, Carbon 111, 113 (2017).

[18] A. Varykhalov, J. Sánchez-Barriga, D. Marchenko, P. Hlawenka, P. S. Mandal, and O. Rader, Nat. Commun. 6, 7610 (2015).

[19] H. Okamoto, J. Phase Equilib. 13, 543 (1992).

[20] S. Vlaic, N. Rougemaille, A. Artaud, V. Renard, L. Huder, J.-L. Rouviére, A. Kimouche, B. Santos, A. Locatelli, V. Guisset et al., J. Phys. Chem. Lett. 9, 2523 (2018).

[21] G. Avvisati, P. Gargiani, D. Lizzit, M. Valvidares, P. Lacovig, C. Petrillo, F. Sacchetti, and M. G. Betti, Appl. Surf. Sci. 527, 146599 (2020).

[22] P. Gargiani, R. Cuadrado, H. B. Vasili, M. Pruneda, and M. Valvidares, Nat. Commun. 8, 699 (2017).
[23] M. Bazarnik, R. Decker, J. Brede, and R. Wiesendanger, Surf. Sci. 639, 70 (2015).

[24] https://sites.google.com/uniroma1.it/nano-surface-physics/.

[25] A. Barla, J. Nicolás, D. Cocco, S. M. Valvidares, J. HerreroMartán, P. Gargiani, J. Moldes, C. Ruget, E. Pellegrin, and S. Ferrer, J. Synchrotron Radiat. 23, 1507 (2016).

[26] G. Avvisati, C. Cardoso, D. Varsano, A. Ferretti, P. Gargiani, and M. G. Betti, Nano Lett. 18, 2268 (2018).

[27] J. Brede, J. Sławińska, M. Abadia, C. Rogero, J. E. Ortega, I. Piquero-Zulaica, J. Lobo-Checa, A. Arnau, and J. I. Cerdá, 2D Mater. 4, 015016 (2016).

[28] See Supplemental Material at http://link.aps.org/supplemental/ 10.1103/PhysRevMaterials.5.014405 for the detailed description of the $\mathrm{C} 1 s$, Ir $4 f_{7 / 2}$, and Fe $2 p_{3 / 2}$ core levels; the LEED spot intensity analysis for $\mathrm{Gr} / \mathrm{Ir}$ (111), Gr/1ML Fe/Ir(111) and $\mathrm{Gr} / 7 \mathrm{ML}$ Fe/Ir(111); the unfolded bands of Gr/1ML-Fe/Ir(111); the spin-resolved pDOS for Gr/1ML-Fe/Ir(111) and Gr/1ML$\mathrm{Co} / \mathrm{Ir}(111)$.

[29] P. Giannozzi, S. Baroni, N. Bonini, M. Calandra, R. Car, C. Cavazzoni, D. Ceresoli, G. L. Chiarotti, M. Cococcioni, I. Dabo et al., J. Phys.: Condens. Matter 21, 395502 (2009).

[30] P. Giannozzi, O. Andreussi, T. Brumme, O. Bunau, M Buongiorno Nardelli, M. Calandra, R. Car, C. Cavazzoni, D. Ceresoli, M. Cococcioni, N. Colonna, I. Carnimeo, A. Dal Corso, S. de Gironcoli, P. Delugas, R. A. DiStasio Jr., A. Ferretti, A. Floris, G. Fratesi, G. Fugallo, R. Gebauer, U. Gerstmann, F. Giustino, T. Gorni, J. Jia, M. Kawamura, H.Y. Ko, A. Kokalj, E. Küçükbenli, M. Lazzeri, M. Marsili, N. Marzari, F. Mauri, N. L. Nguyen, H.-V. Nguyen, A. O. de-la Roza, L. Paulatto, S. Poncé, D. Rocca, R. Sabatini, B. Santra, M. Schlipf, A. P. Seitsonen, A. Smogunov, I. Timrov, T. Thonhauser, P. Umari, N. Vast, X. Wu, and S. Baroni, J. Phys.: Condens. Matter 29, 465901 (2017).

[31] J. P. Perdew and A. Zunger, Phys. Rev. B 23, 5048 (1981).

[32] A. Calloni, G. Bussetti, G. Avvisati, M. S. Jagadeesh, D. Pacilè, A. Ferretti, D. Varsano, C. Cardoso, L. Duò, F. Ciccacci, and M. G. Betti, J. Chem. Phys. 153, 214703 (2020).

[33] C. J. Cramer and D. G. Truhlar, Phys. Chem. Chem. Phys. 11, 10757 (2009).

[34] M. Cococcioni and S. de Gironcoli, Phys. Rev. B 71, 035105 (2005).

[35] V. Popescu and A. Zunger, Phys. Rev. B 85, 085201 (2012).

[36] P. Bonfà, https://bitbucket.org/bonfus/unfold-x.

[37] A. N'Diaye, J. Coraux, T. Plasa, C. Busse, and T. Michely, New J. Phys. 10, 043033 (2008).

[38] M. Scardamaglia, G. Forte, S. Lizzit, A. Baraldi, P. Lacovig, R. Larciprete, C. Mariani, and M. G. Betti, J. Nanopart. Res. 13, 6013 (2011).

[39] C. Busse, P. Lazić, R. Djemour, J. Coraux, T. Gerber, N. Atodiresei, V. Caciuc, R. Brako, A. T. N'Diaye, S. Blügel, J. Zegenhagen, and T. Michely, Phys. Rev. Lett. 107, 036101 (2011).

[40] S. Grimme, J. Comput. Chem. 27, 1787 (2006).

[41] Note that the DFT-D2 correction for van der Walls interactions has been reparametrized in these works.

[42] G. Avvisati, S. Lisi, P. Gargiani, A. Della Pia, O. De Luca, D. Pacilè, C. Cardoso, D. Varsano, D. Prezzi, A. Ferretti, and M. G. Betti, J. Phys. Chem. C 121, 1639 (2017).

[43] V. L. Moruzzi, P. M. Marcus, K. Schwarz, and P. Mohn, Phys. Rev. B 34, 1784 (1986). 
[44] G. Moulas, A. Lehnert, S. Rusponi, J. Zabloudil, C. Etz, S. Ouazi, M. Etzkorn, P. Bencok, P. Gambardella, P. Weinberger, and H. Brune, Phys. Rev. B 78, 214424 (2008).

[45] I. Pletikosić, M. Kralj, P. Pervan, R. Brako, J. Coraux, A. T. N'Diaye, C. Busse, and T. Michely, Phys. Rev. Lett. 102, 056808 (2009).

[46] M. Kralj, I. Pletikosić, M. Petrović, P. Pervan, M. Milun, A. T. N'Diaye, C. Busse, T. Michely, J. Fujii, and I. Vobornik, Phys. Rev. B 84, 075427 (2011).

[47] Y. Dedkov and E. Voloshina, J. Phys.: Condens. Matter 27, 303002 (2015).

[48] Note that comparing ARPES data with KS-DFT calculations absolute quantitative agreement in the position of the features cannot be expected.

[49] S. Heinze, K. von Bergmann, M. Menzel, J. Brede, A. Kubetzka, R. Wiesendanger, G. Bihlmayer, and S. Blügel, Nat. Phys. 7, 713 (2011).

[50] K. von Bergmann, S. Heinze, M. Bode, G. Bihlmayer, S. Blügel, and R. Wiesendanger, New J. Phys. 9, 396 (2007).
[51] K. von Bergmann, S. Heinze, M. Bode, E. Y. Vedmedenko, G. Bihlmayer, S. Blügel, and R. Wiesendanger, Phys. Rev. Lett. 96, 167203 (2006).

[52] K. von Bergmann, M. Menzel, A. Kubetzka, and R. Wiesendanger, Nano Lett. 15, 3280 (2015).

[53] J. Grenz, A. Köhler, A. Schwarz, and R. Wiesendanger, Phys. Rev. Lett. 119, 047205 (2017).

[54] J. Brede, N. Atodiresei, V. Caciuc, M. Bazarnik, A. Al-Zubi, S. Blügel, and R. Wiesendanger, Nat. Nanotechnol. 9, 1018 (2014).

[55] B. T. Thole, P. Carra, F. Sette, and G. van der Laan, Phys. Rev. Lett. 68, 1943 (1992).

[56] P. Carra, B. T. Thole, M. Altarelli, and X. Wang, Phys. Rev. Lett. 70, 694 (1993).

[57] S. Schumacher, T. O. Wehling, P. Lazić, S. Runte, D. F. Förster, C. Busse, M. Petrović, M. Kralj, S. Blügel, N. Atodiresei, V. Caciuc, and T. Michely, Nano Lett. 13, 5013 (2013).

[58] S. Schumacher, F. Huttmann, M. Petrović, C. Witt, D. F. Förster, C. Vo-Van, J. Coraux, A. J. Martínez-Galera, V. Sessi, I. Vergara, R. Rückamp, M. Grüninger, N. Schleheck, F. Meyer zu Heringdorf, P. Ohresser, M. Kralj, T. O. Wehling, and T. Michely, Phys. Rev. B 90, 235437 (2014). 